training and enhance the career development of future generations of clinical and translational science researchers. OBJECTIVES/GOALS: In the field of clinical and translational science, the career trajectory and definition of Junior Investigators (JIs) vary greatly. This study aims to investigate JI characteristics, training, and support that contribute to career development at the University of California Los Angeles (UCLA) Clinical and Translation Science Institute (CTSI). METHODS/STUDY POPULATION: Every 18 months, the UCLA CTSI administers the Longitudinal Scientific Achievement Survey, which collects information on the predictors of scientific productivity and impact. In 2018, a special supplement was added to survey JIs who received CTSA support between 2011 and 2017 ( $n=305)$, including questions on knowledge, use, and effectiveness of CTSA specific support, barriers and facilitators of research, scientific productivity, and perceived scientific impact. A literary analysis was conducted to explore previous categorizations of JIs. The JIs in our sample conducted bench to bedside, population and policy research at our four partner sites. Bivariate and logistic regression analysis were conducted to examine the significant predictors of a new grant award attributed to the CTSA support/services. RESULTS/ANTICIPATED RESULTS: The survey response rate was $82 \%(n=250)$. Respondents include core voucher co-investigators, enrollees in the Training Program in Translational Science, and $\mathrm{K}$ - and K-to-R workshop participants. Bivariate results showed new grant awardees significantly more likely to have the following characteristics: physician scientist with an $\mathrm{MD}$ and $\mathrm{PhD}(47 \%)$, pilot grant awardee (42\%), core voucher awardee (49\%), four or more types of CTSI support (48\%), prior affiliation with an NIH institute/center other than NCATS (42\%), and reported at least one impact in science, health, and/or the community (72\%). Multivariate results showed that investigators with a prior core voucher award, a prior NIH affiliation, or reported one or more impacts were the strongest predictors of obtaining a new grant (each with $\mathrm{OR}>=4.0$ ). DISCUSSION/SIGNIFICANCE OF FINDINGS: The most successful investigators consulted with NIH program officers and received feedback on their research plans and methods. Sufficient funding is crucially important to research progression. In our CTSA hub, vouchers and grants to initiate new studies or offset costs of existing research are consistent predictors of new extramural funding.

75202

\section{The New Normal: A Virtual Summer Foundations in Research}

Adriana Morales Gomez, Kit Knier, Joanna Yang Yowler, Chris Pierret and Linda M. Scholl

${ }^{1}$ Mayo Clinic Graduate School of Biomedical Sciences, Mayo Clinic, Rochester, MN, USA, ${ }^{2}$ Mayo Clinic Medical Scientist Training Program, Mayo Clinic, Rochester, MN, USA, ${ }^{3}$ Department of Research, Mayo Clinic, Jacksonville, FL, USA, ${ }^{4}$ Department of Biochemistry and Molecular Biology, Mayo Clinic, Rochester, MN, USA and ${ }^{5}$ Office of Applied Scholarship and Education Science, Mayo Clinic College of Medicine, Mayo Clinic, Rochester, MN, USA

ABSTRACT IMPACT: The Summer Foundation on Research gave undergraduate students the opportunity to do research despite the new normal - COVID-19 pandemic. OBJECTIVES/GOALS: The COVID-19 pandemic prevented domestic and international undergraduate students from attending in-person Mayo Clinic Summer Undergraduate Research Programs. Mayo decided to redesign this program as a virtual, 4-week Summer Foundations in Research
(SFIR) program. The goal of this program was to give students a scientific research experience. METHODS/STUDY POPULATION: The SFIR included an Introduction to Experimental Design, Dialogue methodology for communicating science, scientific mentoring, asynchronous online modules and a Resiliency component. Evaluations of the program were undertaken to gather feedback for program improvement and to assess the educational and mental health impact on participants. These evaluations asked student to rate each section of the program. Additionally, students were encouraged to provide their own comments and feedback. Statistical analysis of quantitative data was performed using excel. The qualitative data was studied using the identification, analysis and interpretation of patterns method per the student's comments on each of the questions addressed in the survey. RESULTS/ANTICIPATED RESULTS: These evaluations revealed positive outcomes across program components: $66 \%$ of the participants found the Resiliency component extremely worthwhile, $80 \%$ of participants liked the experimental design and $70 \%$ liked the educational courses. Qualitative data showed that mentor/mentee interactions were highly valued, and both participants and faculty suggested increasing the amount of time devoted to these interactions. Small group discussions gave students the opportunity to get to know other peers and encouraged further discussions about science and the community. Participants suggested minor improvements to the program, such as re-creating the online modules specific for undergraduate students, increasing 1to-1 and small group's discussion, and increasing the length of the program. DISCUSSION/SIGNIFICANCE OF FINDINGS: Despite the quick pivot of the SFIR program, the re-design and new format supported the development of participants' resilience skills and training as future scientists during a particularly challenging time. Mayo is committed to continuing this program as an early step in a pathway to careers in research.

\section{Precision Medicine}

74957

Utilizing 3D Printing to Assist Planning of Percutaneous/ Endovascular Procedures in Interventional Radiology

Lucas Richards, Shiv Dalla, Carissa Walter and Aaron Rohr University of Kansas Medical Center

ABSTRACT IMPACT: We plan to measure the impact of integrating $3 \mathrm{D}$ printed models in the planning process of endovascular procedures with the goal of making a case for using this resource more often. OBJECTIVES/GOALS: To measure the impact of using 3D printed models of patient specific anatomy for pre-procedure planning and as an intra-procedure reference. Impact will be measured by: a. Radiation exposure ; b. Contrast dosage; c. Fluoroscopy time; d. Time to procedural completion; e. 'Attempts at access,' when applicable to the procedure METHODS/STUDY POPULATION: Retrospective data will be collected on every patient that received one of prostate artery embolism, transjugular intrahepatic portosystemic shunt placement, or endovascular stent repair in the 3 years prior to the first prospective case. An attempt will be made to create a procedure planning model for every patient that receives one of the three procedures of interest in the 5 months following the first prospective case and those that have a model included in their procedure planning process will be included as part of the experimental group. 